\title{
Effects of Aspirin, Nonsteroidal Anti-inflammatory Drugs, Statin, and COX2 Inhibitor on the Developments of Urological Malignancies: A Population-Based Study with 10-Year Follow-up Data in Korea
}

\author{
Minyong Kang, $\mathrm{MD}, \mathrm{PhD}$ \\ Ja Hyeon Ku, MD, PhD² \\ Cheol Kwak, MD, PhD² \\ Hyeon Hoe Kim, MD, PhD² \\ Chang Wook Jeong, MD, PhD²
}

${ }^{1}$ Department of Urology, Samsung Medical Center, Sungkyunkwan University School of Medicine, Seoul, ${ }^{2}$ Department of Urology, Seoul National University Hospital,

Seoul, Korea
Correspondence: Chang Wook Jeong, MD, PhD Department of Urology, Seoul National University Hospital, 101 Daehak-ro, Jongno-gu,

Seoul 03080, Korea

Tel: 82-2-2072-0361

Fax: 82-2-742-4665

E-mail:drboss@gmail.com

Received May 22, 2017

Accepted October 25, 2017

Published Online October 27, 2017

\section{Purpose}

The purpose of this study was to determine the impact of aspirin, nonsteroidal anti-inflammatory drugs (NSAIDs), statin, and cyclooxygenase 2 (COX-2) inhibitor on the development of kidney, prostate, and urothelial cancers by analyzing the Korean National Health Insurance Service-National Sample Cohort (NHIS-NSC) database.

\section{Materials and Methods}

Among a representative sample cohort of 1,025,340 participants in NHIS-NSC database in 2002 , we extracted data of 799,850 individuals who visited the hospital more than once, and finally included 321,122 individuals aged 40 and older. Following a 1-year washout period between 2002 and 2003, we analyzed 143,870 (male), 320,861 and 320,613 individuals for evaluating the risk of prostate cancer, kidney cancer and urothelial cancer developments, respectively, during 10-year follow-up periods between 2004 and 2013. The medication group consisted of patients prescribed these drugs more than $60 \%$ of the time in 2003. To adjust for various parameters of the patients, a multivariate Cox regression model was adopted.

\section{Results}

During 10-year follow-up periods between 2004 and 2013, 9,627 (6.7\%), 1,107 (0.4\%), and 2,121 (0.7\%) patients were diagnosed with prostate cancer, kidney cancer, and urothelial cancer, respectively. Notably, multivariate analyses revealed that NSAIDs significantly increased the risk of prostate cancer (hazard ratio [HR], 1.35). Also, it was found that aspirin $(H R, 1.28)$ and statin $(H R, 1.55)$ elevated the risk of kidney cancer. No drugs were associated with the risk of urothelial cancer.

\section{Conclusion}

In sum, our study provides the valuable information for the impact of aspirin, NSAID, statin, and COX-2 inhibitor on the risk of prostate, kidney, and urothelial cancer development and its survival outcomes.

\section{Introduction}

Aspirin, statins, and nonsteroidal anti-inflammatory drugs (NSAID) are commonly prescribed drugs in the world [1]. Approximately $10 \%$ of the overall population in the United States is prescribed statins, while its prevalence is increasing

\section{Key words}

National Health Insurance Corporation Database,

Prostate neoplasms, Kidney neoplasms, Urothelial cancer, Anti-inflammatory agents 
tric and breast cancer by regulating various signaling pathways, including cyclooxygenase (COX)- 1 and -2, AMP-activated protein kinase, mammalian target of rapamycin, and mitogen-activated protein kinase [3]. Statins (3-hydroxy3-methylglutaryl-coenzyme A reductase inhibitors) have shown potential anti-neoplastic effects via inducing apoptosis, regulating cell cycle disturbance, and suppressing the growth of tumors [4]. It was also demonstrated that longterm use of NSAIDs reduced the risk of colorectal, lung, and breast cancer development by exerting anti-apoptotic and anti-proliferative effects [5].

In urological malignancies, several epidemiological studies presented the beneficial effects of aspirin, NSAIDs, and statins on the risk of cancer development, while other reports suggest no benefits of these drugs. Therefore, the chemopreventive effects of these anti-inflammatory drugs on urological cancers still remain inconclusive [6,7]. Moreover, previous evidence was primarily based on the Western population, which is usually different in genetic and environmental background compared to Asian population, and the possibilities of different responsiveness to the same type of drugs.

We aimed to clarify the impact of aspirin, NSAIDs, statins and COX-2 inhibitors on the risk for kidney, prostate, and urothelial cancers in the Korean population aged 40 and older by analyzing the National Health Insurance (NHI) Corporation database.

\section{Materials and Methods}

In this study, we used the National Health Insurance Service-National Sample Cohort (NHIS-NSC), which is a population-based cohort developed by the National Health Insurance Service (NHIS) in our country [8]. Among a representative sample cohort of 1,025,340 participants in NHISNSC database of 2002, we collected data from 799,850 individuals who visited the hospital more than once in 2002. Of these participants, we only included 321,122 individuals aged 40 and older as a total study population for evaluating the risk of cancer development. We screened data with the code C61, C64, and C65-67, indicating prostate, kidney, and urothelial cancers, respectively, according to the National Center for Health Statistics International Classification of Diseases and Related Health Problems, 10th edition (ICD10). After a 1-year washout period between 2002 and 2003, we excluded patients with pre-existing urological malignancies, such as prostate cancer (PCa) $(\mathrm{n}=1,551)$, kidney cancer $(n=261)$, and urothelial cancer $(n=509)$, before the study period. Among a total of 321,122 persons aged 40 and older, we finally analyzed 143,870 male individuals for evaluating the risk of PCa (group 1), as well as 320,861 and 320,613 individuals for evaluating the risk of kidney cancer (group 2 ) and urothelial cancer (group 3) developments, respectively, during 10-year follow-up periods from January 2004 to December 2013.

We reviewed the baseline demographics of patients as follows: age at diagnosis, insurance eligibility status, Charlson Comorbidity Index (CCI), which is defined as the sum of weights based on predetermined clinical status within the diagnosis year [9], participant's income level, the prescription of anti-inflammatory drugs (aspirin, NSAIDs, statin, and COX2-inhibitor), and the diagnosis with prostate, kidney and urothelial cancers during a 10-year follow-up period. We classified the patients into two groups according to the use of anti-inflammatory drugs (non-user vs. drug user). The drug-using group was defined by their prescription of antiinflammatory drugs more than $60 \%$ of the time in 2003.

We performed Kaplan-Meier analysis for estimating the risk of prostate, kidney or urothelial cancer development over a 10 period since 2003, according to the exposure of aspirin, NSAIDs, statins, or COX-2 inhibitors. A log-rank test was used to determine the significant differences between the drug-using and non-drug using groups. To adjust various parameters of patients, including age distribution, sex, insurance eligibility status, CCI, participant's income level and whether or not drug usage and drug types, the multivariate Cox proportional hazards model was applied to analyze the effects of each agent on the urological cancer occurrence. Two-sided p-values of less than 0.05 were regarded as statistically significant. The SAS software ver. 9.3 (SAS Institute Inc., Cary, NC) was used for statistical analysis.

\section{Ethical statement}

The study was approved by the Institutional Review Board of Seoul National University Hospital (IRB No. E-1608-015781) and performed in accordance with the principles of the Declaration of Helsinki. The informed consent was waived.

\section{Results}

Baseline demographics of the overall population are presented in Table 1. Among the patients extracted from the NHIS-NSC database of 2003, a total of 9,627 (6.7\%), 1,107 $(0.4 \%)$, and 2,121 $(0.7 \%)$ patients were diagnosed with $\mathrm{PCa}$, kidney cancer and urothelial cancer, respectively, during a 10-year follow-up period between January 2004 and December 2013. Among four analyzed drugs, aspirin was most 
Table 1. Baseline demographics of the overall populations for evaluating the risk of prostate cancer, kidney cancer, and urothelial cancer development for 10 years

\begin{tabular}{|c|c|c|c|}
\hline Variable & $\begin{array}{c}\text { Group } 1 \\
\text { (for prostate cancer) } \\
(\mathrm{n}=\mathbf{1 4 3 , 8 7 0 )}\end{array}$ & $\begin{array}{c}\text { Group } 2 \\
\text { (for kidney cancer) } \\
(\mathrm{n}=\mathbf{3 2 0 , 8 6 1 )}\end{array}$ & $\begin{array}{c}\text { Group } 3 \\
\text { (for urothelial cancer) } \\
(\mathrm{n}=320,613)\end{array}$ \\
\hline \multicolumn{4}{|l|}{ Age distribution (yr) } \\
\hline $40-50$ & $61,984(43.1)$ & $131,148(40.9)$ & $131,131(40.9)$ \\
\hline $50-60$ & $37,949(26.4)$ & $81,758(25.5)$ & $81,720(25.5)$ \\
\hline $60-70$ & $29,544(20.5)$ & $66,881(20.8)$ & $66,807(20.8)$ \\
\hline$>70$ & $14,393(10.0)$ & $41,074(12.8)$ & $40,955(12.8)$ \\
\hline \multicolumn{4}{|l|}{ Sex } \\
\hline Female & - & $175,605(54.7)$ & $175,546(54.7)$ \\
\hline Male & $143,870(100)$ & $145,256(45.3)$ & $145,067(45.3)$ \\
\hline \multicolumn{4}{|l|}{ Insurance eligibility status } \\
\hline National Health Insurance & $143,202(99.5)$ & $318,978(99.4)$ & $318,733(99.4)$ \\
\hline Medical aid & $668(0.5)$ & $1,883(0.6)$ & $1,880(0.6)$ \\
\hline \multicolumn{4}{|l|}{ Charlson Comorbidity Index } \\
\hline 0 & $104,198(72.4)$ & $226,731(70.7)$ & $226,654(70.7)$ \\
\hline 1 & $29,152(20.3)$ & $68,758(21.4)$ & $68,714(2.4)$ \\
\hline 2 & $7,617(5.3)$ & $18,605(5.8)$ & $18,519(5.8)$ \\
\hline$\geq 3$ & $2,903(2.0)$ & $6,767(2.1)$ & $6,726(2.1)$ \\
\hline \multicolumn{4}{|l|}{ Participant's income level } \\
\hline $1-4$ & $37,394(26.0)$ & $92,025(28.7)$ & $91,949(28.7)$ \\
\hline $5-8$ & $58,049(40.4)$ & $125,171(39.0)$ & $125,095(39.0)$ \\
\hline $9-10$ & $48,427(33.6)$ & $103,665(32.3)$ & $103,569(32.3)$ \\
\hline \multicolumn{4}{|l|}{ Drug types } \\
\hline Aspirin & $5,652(3.9)$ & $11,838(3.7)$ & $11,835(3.7)$ \\
\hline NSAIDs & $1,305(0.9)$ & 5,192 (1.6) & $5,190(1.6)$ \\
\hline Statin & $1,667(1.2)$ & $4,038(1.3)$ & $4,038(1.3)$ \\
\hline COX-2 inhibitors & $131(0.1)$ & $536(0.2)$ & $535(0.2)$ \\
\hline \multicolumn{4}{|c|}{ No. of cancer development (2003-2013) } \\
\hline Total & $9,622(6.7)$ & $1,107(0.4)$ & $2,121(0.7)$ \\
\hline Person-year (/100,000/yr) & 668.7 & 34.5 & 66.2 \\
\hline \multicolumn{4}{|l|}{ No. of deaths (2003-2013) } \\
\hline All-cause death & $1,413(14.6)$ & $222(20.1)$ & $502(23.6)$ \\
\hline Cancer-specific death & $164(1.7)$ & $81(7.3)$ & $154(7.2)$ \\
\hline
\end{tabular}

Values are presented as number (\%). Overall population included all individuals who were recorded at the National Insurance System in $2003(n=799,850)$, particularly who were aged more than 40 years $(n=321,122)$ as well as excluded those who were already diagnosed with each of the cancers after 1-year wash out period. NSAID, nonsteroidal anti-inflammatory drug; COX2 , cyclooxygenase 2 .

commonly prescribed in patients of group $1(3.9 \%, \mathrm{n}=5,652)$, group $2(3.7 \%, \mathrm{n}=11,838)$, and group $3(3.7 \%, \mathrm{n}=11,835)$, respectively. We further classified the overall population into three groups, such as never medication, partial medication (less than 60\%), and full medication (more than 60\%), and compared the baseline demographics according to the medication types (S1 Table).

We next examined the effects of aspirin, NSAIDs, statin and COX-2 inhibitor on the risk of urological malignancies by adjusting other clinical variables. Results are summarized in Table 2. Of note, NSAID was identified as an independent predictor for the increased risk of PCa (hazard ratio [HR], 1.35; 95\% confidence interval [CI], 1.14 to 1.58 ), in addition to older age, poor CCI and higher participant's income level. For kidney cancer, aspirin (HR, 1.28; 95\% CI, 1.01 to 1.64) and statin (HR, 1.55; 95\% CI, 1.08 to 2.23 ) remained as the predictive factors for the development of cancer, as well as for older age, male sex, and poor $\mathrm{CCI}$ and higher participant's income 


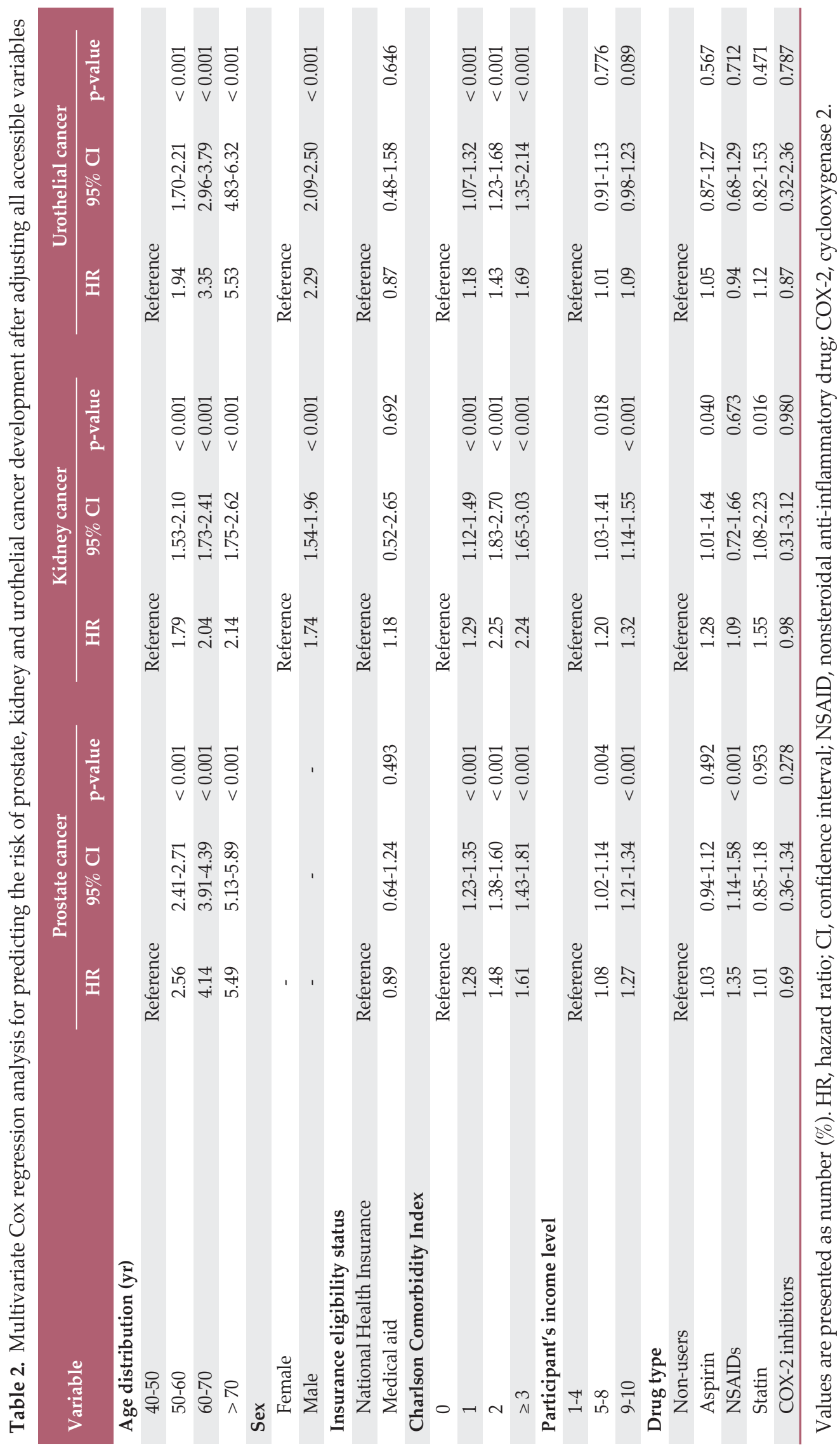




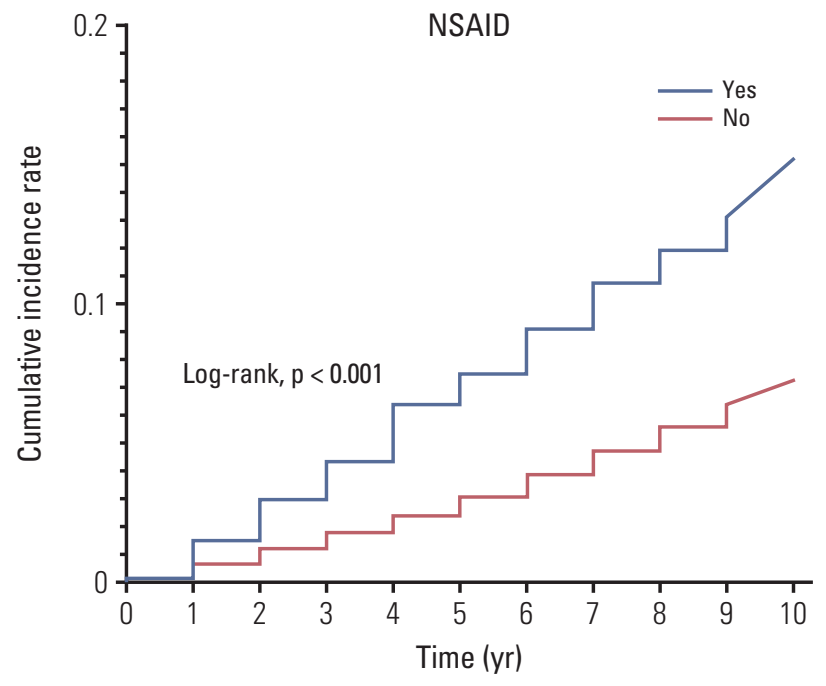

Fig. 1. Kaplan-Meier analysis for estimating the risk of prostate cancer development over 10 years (2003-2013) according to the prescription of nonsteroidal anti-inflammatory drugs (NSAIDs; yes vs. no). Log-rank test was used to define the statistical differences between the two groups.

level. However, no drugs were associated with the risk of urothelial cancer development, whereas older age, male sex, and poor CCI were proved as the significant predictors for cancer occurrence.

These results were represented by the Kaplan-Meier analysis for estimating the risk of prostate and kidney cancer according to the type of drugs used. As shown in Fig. 1, patients who take NSAIDs demonstrated a remarkably higher cumulative incidence rate of PCa development compared to those who did not take NSAIDs. When we classified the overall population into three groups (non-user, partial, and full medication), patients with full medication showed a higher risk of PCa development than other two groups (S2 Fig.). In addition, Fig. 2 presents those patients who take aspirin or statins, revealing significantly higher cumulative incidence rates of kidney cancer developments compared to their counterparts who were not prescribed aspirin or statins. We also confirmed that patients with full medication of aspirin or statins had a higher risk of kidney cancer development than partial medication and non-user groups (S3 Fig.).

Among patients diagnosed with prostate, kidney cancer and urothelial cancer, respectively, during a 10-year followup period, the numbers of all-cause and cancer-specific deaths, respectively, are as follows: 1,413 (14.6\%) and 164 $(1.7 \%)$ in prostate cancer; $222(20.1 \%)$ and $81(7.3 \%)$ in kidney cancer; $502(23.6 \%)$ and $154(7.2 \%)$ in urothelial cancer (Table 1). More importantly, Kaplan-Meier survival analyses revealed that patients with full medication of NSAID showed worse survival outcomes compared to other two medication groups in patients with PCa (S4A Fig.). However, among
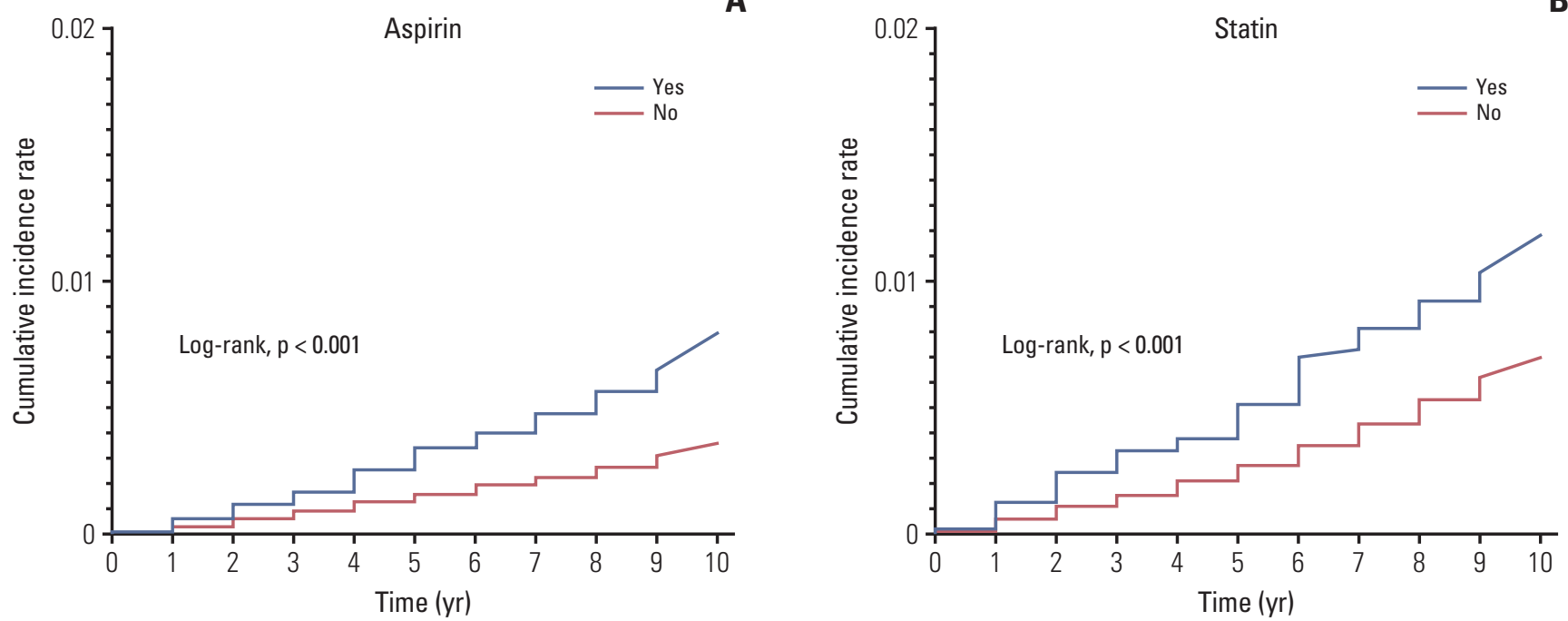

Fig. 2. Kaplan-Meier analysis for estimating the risk of kidney cancer development over 10 years (2003-2013) according to the prescription of aspirin (A) and statin (yes vs. no) (B), respectively. Log-rank tests were used to define the statistical differences between the two groups. 
kidney cancer, patients with full medication of aspirin, not statins, had worse overall survival rates than partial medication and non-user groups (S4B and S4C Fig.). For cancer-specific survivals, there were no differences according to drug types in all cancer types (data not shown).

\section{Discussion}

To the best of our knowledge, our study was the first to analyze the NHIS-NSC database of South Korea for determining the effects of aspirin, NSAIDs, statin and COX-2 inhibitor on the risk of developing urological malignancies (prostate, kidney and urothelial cancers). The key finding of the current study was that NSAIDs were associated with increased risk of PCa (HR, 1.35), and aspirin (HR, 1.28) and statin (HR, 1.55), were associated with a higher risk of kidney cancer in the multivariate Cox regression analyses. Conversely, no drugs were identified as the independent predictors for the risk of cancer occurrence in urothelial cancer.

In contrast to our findings, preclinical studies have provided evidence on the inhibitory effects of NSAIDs on PCa development and progression [10]. Mechanistically, NSAIDs inhibited cancer cell growth and induced apoptotic cell death via suppression of AKT signaling pathway and cell cycle (G1) arrest in LNCaP and PC3 cells [11]. However, results of other clinical studies are conflicting and inconsistent with this. A nationwide study by Skriver et al. [12] analyzed 35,600 patients who were diagnosed with PCa between 2000 and 2012, and revealed that the exposure of NSAIDs significantly increased the risk of localized PCa development (HR, 1.13; 95\% CI, 1.10 to 11.15). In addition, a Finnish Prostate Cancer Screening Trial examined the risk of PCa occurrence in a total of 78,615 PCa patients, and confirmed that the risk was significantly elevated in the population that was currently receiving NSAIDs (HR, 1.45). This was not seen in those patients with a previous use of NSAIDs [13]. However, a recent meta-analysis included 31 observational studies and revealed that the use of any NSAIDs was not associated with the risk reduction of overall PCa (HR, $0.90 ; 95 \% \mathrm{CI}, 0.75$ to 1.07) and advanced PCa (HR, 0.86 ; $95 \%$ CI, 0.52 to 1.40$)$ incidence [7]. Taken together, further well-designed studies are required to determine the association between the use of NSAIDs and the risk of PCa development.

For kidney cancer, Bosetti et al. [6] performed a metaanalysis with 10 case-control and seven cohort studies, showing no association between the regular use of aspirin and the risk of kidney cancer diagnosis (HR, 1.14; $95 \% \mathrm{CI}, 0.95$ to 1.37). Choueiri et al. [14] reported the largest meta-analysis, including 20 studies with 8,420 kidney cancer patients, and found that the use of aspirin was not identified as an independent predictor for kidney cancer development (HR, 1.10; $95 \%$ CI, 0.95 to 1.28). However, the exposure of aspirin was associated with a significantly increased risk of kidney cancer development in the reports from non-U.S. countries (HR, 1.17; $95 \%$ CI, 1.04 to 1.33), consistent with our findings in the present study. Conversely, there was no association between the use of aspirin and an increased risk of kidney cancer (HR, $1.05 ; 95 \%$ CI, 0.81 to 1.36 ) in the studies from the U.S. More interestingly, the authors also found that regular or any use of NSAIDs was independently associated with an elevated risk of the kidney cancer development (HR, 1.25; 95\% CI, 1.06 to 1.46). Although aspirin proves itself as an effective agent to prevent the development and recurrence of colorectal cancer, it may not be helpful in kidney cancer and can even be harmful [15]. These conflicting results of aspirin use in different types of cancer are due to the differences in tissue-specific mechanisms on cancer prevention or the differences in the underlying mechanisms of carcinogenesis according to the type of malignancies.

Many in vitro and in vivo studies have revealed the anticancer effects of statins to inhibit growth and the progression of cancer cells in various type of malignancies, such as lung, colorectal, prostate and kidney cancers [16]. For example, Fang et al. [17] reported that simvastatin significantly repressed cell growth of human renal cell carcinoma cells (A498 and 786-O) via inhibiting the AKT, mammalian target of rapamycin, ERK, and JAK2/STAT3 signaling pathways. The authors revealed that simvastatin treatment reduced growth, migration and invasion of tumors in renal cell carcinoma xenograft models [17]. Moreover, the Nurses' Health Study and Health Professionals Follow-Up Study with two prospective cohorts provided evidence that the use of statin was significantly associated with a decreased risk of renal cell carcinoma development, particularly in patients without the history of hypertension [18]. However, our results suggested that there is a potential risk of kidney cancer development in statin users. Similarly, a comprehensive metaanalysis reviewing 12 articles showed that the overall and long-term use of statins did not increase the risk of kidney cancer, respectively [19]. A nationwide case-control study in Denmark also demonstrated that long-term prescription of statins did not increase the risk of renal cell carcinoma (HR, 1.06; $95 \%$ CI, 0.91 to 1.23 ) [20]. In this regard, the clinical results need to be further investigated to acquire the more convincing evidence on the chemopreventive effects of statin use in kidney cancer.

Interestingly, other key findings of the current study were that no drugs were associated with the risk of urothelial cancer development. In the pooled analysis including three large prospective trials with 508,842 patients, the regular exposure of NSAIDs significantly reduced bladder cancer risk, partic- 
ularly in the population of non-smokers, while the use of aspirin was not associated with the risk of bladder cancer development [21]. Although a few observational studies showed a protective effect of aspirin on bladder cancer development, a meta-analysis for three case-control and six cohort studies also highlighted no association between the regular use of aspirin and the risk of bladder cancer (HR, $0.95 ; 95 \% \mathrm{CI}, 0.83$ to 1.07 ) [6]. In a population-based case-control study with 325 patients with bladder cancer and 1,300 healthy controls, neither the beneficial nor the harmful impact of statins was confirmed on the risk of bladder cancer development [22]. Additionally, Zhang et al. [23] performed a meta-analysis by studying a total of 13 studies and suggested that a statin prescription did not influence the risk of bladder cancer occurrence in either the overall population or subgroup analysis.

We should discuss the several limitations of the current study. First, despite the large study population, the retrospective nature of the study is the critical drawback, including the susceptibility of selection bias, information bias with insufficient data, frequently missing data on potential confounding factors, and the risk of follow-up loss. Second, only limited clinical information could be retrieved from the national database and adjustments of well-known prognosticators, such as smoking and obesity, were not included in the multivariate analyses. Third, although pathologic features of each urological cancer are also crucial for evaluating oncological outcomes, no information of pathology was acquired from the National Database. Fourth, although the effects of chemoprevention can depend on the overall accumulation of drugs, we could not obtain the detailed information of drug prescription, including dose and duration. Finally, the disagreement between the medical records, such as pathologic report, and the claims code from insurance database can be existed, because the NHIS-NSC database was not established for research purpose but for medical service claims and reimbursement [24]. This has been pointed out as one of the disadvantages of study by using the NHIS-NSC cohort [24]. In this regard, there is a potential risk of selection bias when study population is selected only through the diagnostic code in claims. For example, the right participant can be missing or the wrong participant can be selected. To minimize the risk of this unavoidable bias, we should try to validate the diagnostic accuracy of the claim codes by using not only the diagnosis but also the history of surgery, the medical cost for the cancer code, and prescription of each cancer related drugs.

In conclusion, our results from the Korean NHIS-NSC database revealed that NSAIDs increased the risk of PCa, and the use of aspirin and statin elevated the risk of kidney cancer occurrence, whereas no drugs were associated with urothelial cancer risk. Additionally, the full medication of NSAIDs and aspirin diminished the overall survival, not cancer-specific survival, in patients with prostate and kidney cancer, respectively. We provide the valuable information for the effects of aspirin, NSAID, statin and COX-2 inhibitor on the risk of prostate, kidney and urothelial cancer development and associated survival outcomes in Korea. Therefore, we believe that the present findings should be considered when prescribing anti-inflammatory drugs in individuals aged over 40 years.

\section{Electronic Supplementary Material}

Supplementary materials are available at Cancer Research and Treatment website (http:// www.e-crt.org).

\section{Conflicts of Interest}

Conflict of interest relevant to this article was not reported.

\section{Acknowledgments}

This study was supported by the research grant funded by the Korean Urologic Oncology Society (Grant number: 2015-02). This research was also supported by a grant of the Korea Health Technology R\&D Project through the Korea Health Industry Development Institute (KHIDI), funded by the Ministry of Health \& Welfare, Republic of Korea (Grant number: HI17C0025).

\section{References}

1. Kaufman DW, Kelly JP, Rosenberg L, Anderson TE, Mitchell AA. Recent patterns of medication use in the ambulatory adult population of the United States: the Slone survey. JAMA. 2002;287:337-44.

2. Iacobone M, Citton M, Viel G, Boetto R, Bonadio I, Mondi I, et al. Adrenalectomy may improve cardiovascular and metabolic impairment and ameliorate quality of life in patients with adrenal incidentalomas and subclinical Cushing's syndrome. Surgery. 2012;152:991-7.

3. Patrignani P, Patrono C. Aspirin and cancer. J Am Coll Cardiol. 2016;68:967-76.

4. Matusewicz L, Meissner J, Toporkiewicz M, Sikorski AF. The 
effect of statins on cancer cells: review. Tumour Biol. 2015;36: 4889-904.

5. Rayburn ER, Ezell SJ, Zhang R. Anti-inflammatory agents for cancer therapy. Mol Cell Pharmacol. 2009;1:29-43.

6. Bosetti C, Rosato V, Gallus S, La Vecchia C. Aspirin and urologic cancer risk: an update. Nat Rev Urol. 2012;9:102-10.

7. Liu Y, Chen JQ, Xie L, Wang J, Li T, He Y, et al. Effect of aspirin and other non-steroidal anti-inflammatory drugs on prostate cancer incidence and mortality: a systematic review and metaanalysis. BMC Med. 2014;12:55.

8. Lee J, Lee JS, Park SH, Shin SA, Kim K. Cohort profile: The National Health Insurance Service-National Sample Cohort (NHIS-NSC), South Korea. Int J Epidemiol. 2017;46:e15.

9. Charlson ME, Pompei P, Ales KL, MacKenzie CR. A new method of classifying prognostic comorbidity in longitudinal studies: development and validation. J Chronic Dis. 1987;40: 373-83.

10. Ishiguro H, Kawahara T. Nonsteroidal anti-inflammatory drugs and prostatic diseases. Biomed Res Int. 2014;2014: 436123.

11. Kulp SK, Yang YT, Hung CC, Chen KF, Lai JP, Tseng PH, et al. 3-phosphoinositide-dependent protein kinase-1/ Akt signaling represents a major cyclooxygenase-2-independent target for celecoxib in prostate cancer cells. Cancer Res. 2004;64: 1444-51.

12. Skriver C, Dehlendorff C, Borre M, Brasso K, Sorensen HT, Hallas J, et al. Low-dose aspirin or other nonsteroidal antiinflammatory drug use and prostate cancer risk: a nationwide study. Cancer Causes Control. 2016;27:1067-79.

13. Veitonmaki T, Murtola TJ, Talala K, Taari K, Tammela T, Auvinen A. Non-steroidal anti-inflammatory drugs and cancer death in the Finnish Prostate Cancer Screening Trial. PLoS One. 2016;11:e0153413.

14. Choueiri TK, Je Y, Cho E. Analgesic use and the risk of kidney cancer: a meta-analysis of epidemiologic studies. Int J Cancer.
2014;134:384-96.

15. Tougeron D, Sha D, Manthravadi S, Sinicrope FA. Aspirin and colorectal cancer: back to the future. Clin Cancer Res. 2014;20: 1087-94.

16. Altwairgi AK. Statins are potential anticancerous agents (review). Oncol Rep. 2015;33:1019-39.

17. Fang Z, Tang Y, Fang J, Zhou Z, Xing Z, Guo Z, et al. Simvastatin inhibits renal cancer cell growth and metastasis via AKT/mTOR, ERK and JAK2/STAT3 pathway. PLoS One. 2013;8:e62823.

18. Liu W, Choueiri TK, Cho E. Statin use and the risk of renal cell carcinoma in 2 prospective US cohorts. Cancer. 2012;118:797803.

19. Zhang XL, Liu M, Qian J, Zheng JH, Zhang XP, Guo CC, et al. Statin use and risk of kidney cancer: a meta-analysis of observational studies and randomized trials. $\mathrm{Br} \mathrm{J}$ Clin Pharmacol. 2014;77:458-65.

20. Pottegard A, Clark P, Friis S, Hallas J, Lund L. Long-term use of statins and risk of renal cell carcinoma: a population-based case-control study. Eur Urol. 2016;69:877-82.

21. Daugherty SE, Pfeiffer RM, Sigurdson AJ, Hayes RB, Leitzmann M, Schatzkin A, et al. Nonsteroidal antiinflammatory drugs and bladder cancer: a pooled analysis. Am J Epidemiol. 2011;173:721-30.

22. Kuo CC, Chiu HF, Lee IM, Kuo HW, Lee CT, Yang CY. Statin use and the risk of bladder cancer: a population-based casecontrol study. Expert Opin Drug Saf. 2012;11:733-8.

23. Zhang XL, Geng J, Zhang XP, Peng B, Che JP, Yan Y, et al. Statin use and risk of bladder cancer: a meta-analysis. Cancer Causes Control. 2013;24:769-76.

24. Seong SC, Kim YY, Khang YH, Park JH, Kang HJ, Lee H, et al. Data resource profile: the National Health Information Database of the National Health Insurance Service in South Korea. Int J Epidemiol. 2017;46:799-800. 